\title{
(2) \\ Banana Cultivation in South Asia and East Asia: A review of the evidence from archaeology and linguistics
}

\author{
Dorian Q. Fuller and Marco Madella
}

\section{Research}

\begin{abstract}
South Asia provides evidence for introduced banana cultivars that are surprisingly early in the Indus Valley but late elsewhere in India. Although phytolith data are still limited, systematic samples from fourteen sites in six regions suggest an absence of bananas from most of Neolithic/Chalcolithic South Asia, but presence in part of the Indus valley. Evidence from textual sources and historical linguistics from South Asia and from China suggest the major diffusion of banana cultivars was in the later Iron Age or early historic period, c. 2000 years ago. Nevertheless Harappan period phytolith evidence from Kot Diji, suggests some cultivation by the late third or early second millennium B.C., and the environmental context implies hybridization with Musa balbisiana Colla had already occurred. Evidence of wild banana seeds from an early Holocene site in Sri Lanka probably attests to traditions of utilisation of $M$. balbisiana, a plausible area for hybridization with cultivated Musa acuminata Colla bananas, perhaps already being moved by the later third millennium B.C. Hybridization here, and/or in the New Guinea area now seems more plausible than hybridization in northern Southeast Asia (from Burma through Eastern India) as Simmonds had hypothesized.
\end{abstract}

\section{Introduction}

Neither India nor China was a likely area of banana domestication, yet these regions help us to constrain aspects of the historical geography of early banana cultivation and spread. With long written traditions, our knowledge of bananas in both India and China can be informed by historical sources for much of the past 2000 years. In addition, India and China geographically define roughly the western and northern limits of wild Musa spp. in Asia, and especially the $\mathrm{B}$ genome source of hybrid bananas, Musa balbisiana Colla. In this paper we will start by reviewing the distribution of wild Musa spp. in India and China at the present and what can be suggested for the early and mid Holocene from palaeoecological reconstructions. Archaeological evidence for bananas in these regions remains very limited. Our purpose in this contribution is to situate those few data points of prehistoric banana phytoliths and seeds within the history of appropriate sampling (e.g., for phytoliths) that might have provided evidence for bananas, thus highlighting the potential for more intensive future efforts. We also review some evidence from historical linguistics and textual historical sources on the early history of bananas in India and China.

\section{Cultivated and Wild \\ Bananas in South Asia}

There is hardly a cottage in India that has not its grove of plantains. The natives live almost upon them; and the stems of the plantain, laden with their branches of fruit, are invariably placed at the entrance of their houses during their marriage or other festivals, appropriate emblems of plenty and fertility. (Drury 1873:301)

\section{Correspondence}

Dorian Q. Fuller, Institute of Archaeology, University College London, UNITED KINGDOM.

d.fuller@ucl.ac.uk

Marco Madella, ICREA/Institució Milà i Fontanals, Spanish National Research Council (CSIC), SPAIN.

marco.madella@icrea.es

Ethnobotany Research \& Applications 7:333-351 (2009)

Published: July 30, 2009 


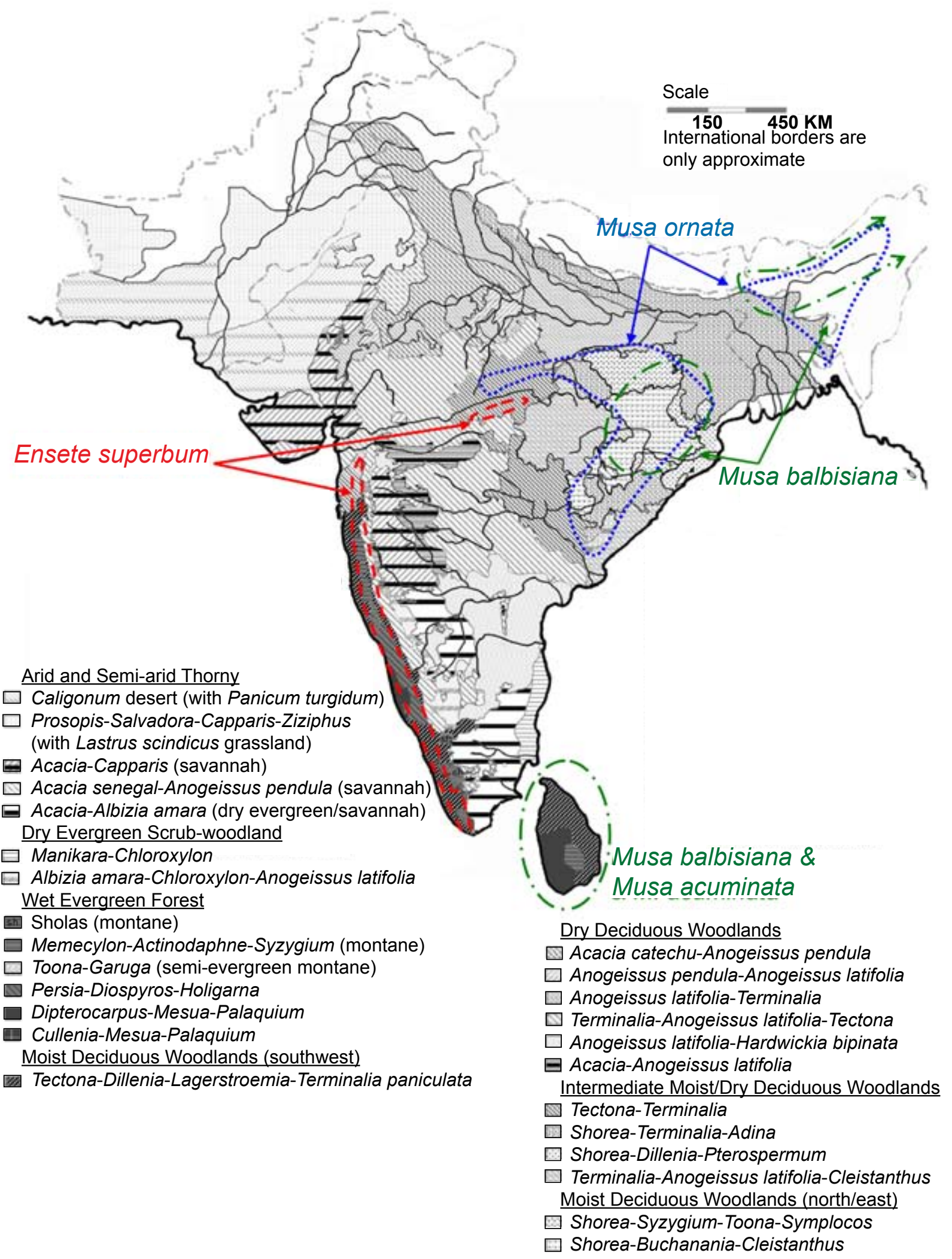

Figure 1. Distribution of wild Musaceae in Indian core region in relation to potential vegetation zones. Base map from Asouti and Fuller (2008). Notes: Temperate and Himalayan types excluded. Sri Lanka vegetation zones are inaccurate, and only indicative of approximate bioclimatic equivalence to South Indian vegetation. 


\section{Fuller \& Madella - Banana Cultivation in South Asia and East Asia: A review of the evidence from archaeology and linguistics}

Bananas, including both starchy and sweet varieties, are a regular component of South Asian landscapes of the present and recent past. A few banana plants are indeed a common element in most house gardens, but unlike in parts of Indonesia, New Guinea or Africa where bananas are a staple food, in South Asian subsistence they are not. There is no reason to regard bananas as much more than a regular supplement to diets focused on grain staples, including rice, wheat, millets and pulses. Larger production of bananas occurs in the peninsular region, eastern India, Gujarat (ICAR 1980), and Sindh (Pakistan), although plantations of sweet varieties of bananas are apparently a very recent (twentieth century) introduction in Sindh (Rahaman 1993). Pure forms of $M$. balbisiana (BB) may be cultivated in South India, where the leaves are used as plates (Ambasta et al. 1986).

Wild Musaceae have a sporadic distribution in South Asia; they are restricted to a few vegetation zones with sufficient water and locally suitable micro-habitats. First it must be noted that there are few wild species, of which Ensete superbum W.J. Tutcher is the main one, through most of India. Figure 1 indicates the reported distribution of wild Musaceae in relation to vegetation zones in the core of India. In the peninsula, E. superbum occurs throughout the hills of the Western Ghats in evergreen and moist deciduous forests as far north as Nagar Haveli/ Dadra (Fischer 1928, Sharma et al. 1984, Sharma et al. 1996, Sharma \& Singh 2001), growing on steep slopes and rocky cliffs (Figure 2), thus occupying the same niche as $M$. balbisiana in eastern India or Sri Lanka (see Haines 1921-1925). This species, Ensete superbum, has apparent relict distributions through some of the central Indian hill ranges, reported as far east as Hoshnagbad, Madhya Pradesh, along the southern side of the Narmada valley (Singh et al. 2001). Ensete superbum is reported to be eaten; its inflorescence may be a vegetable and its young fruits may be pickled (Ambasta et al. 1986). In Sri Lanka Musa acuminata Colla is reported (Hooker 1872-1897), but it appears to be absent from treatises of southern Indian flora (e.g., Fischer 1928). Wild Musaceae (a single species, E. superbum) are restricted to the west-facing Western Ghats, where rainfall is high and they are not reported from the eastern side in central Tamil Nadu or in

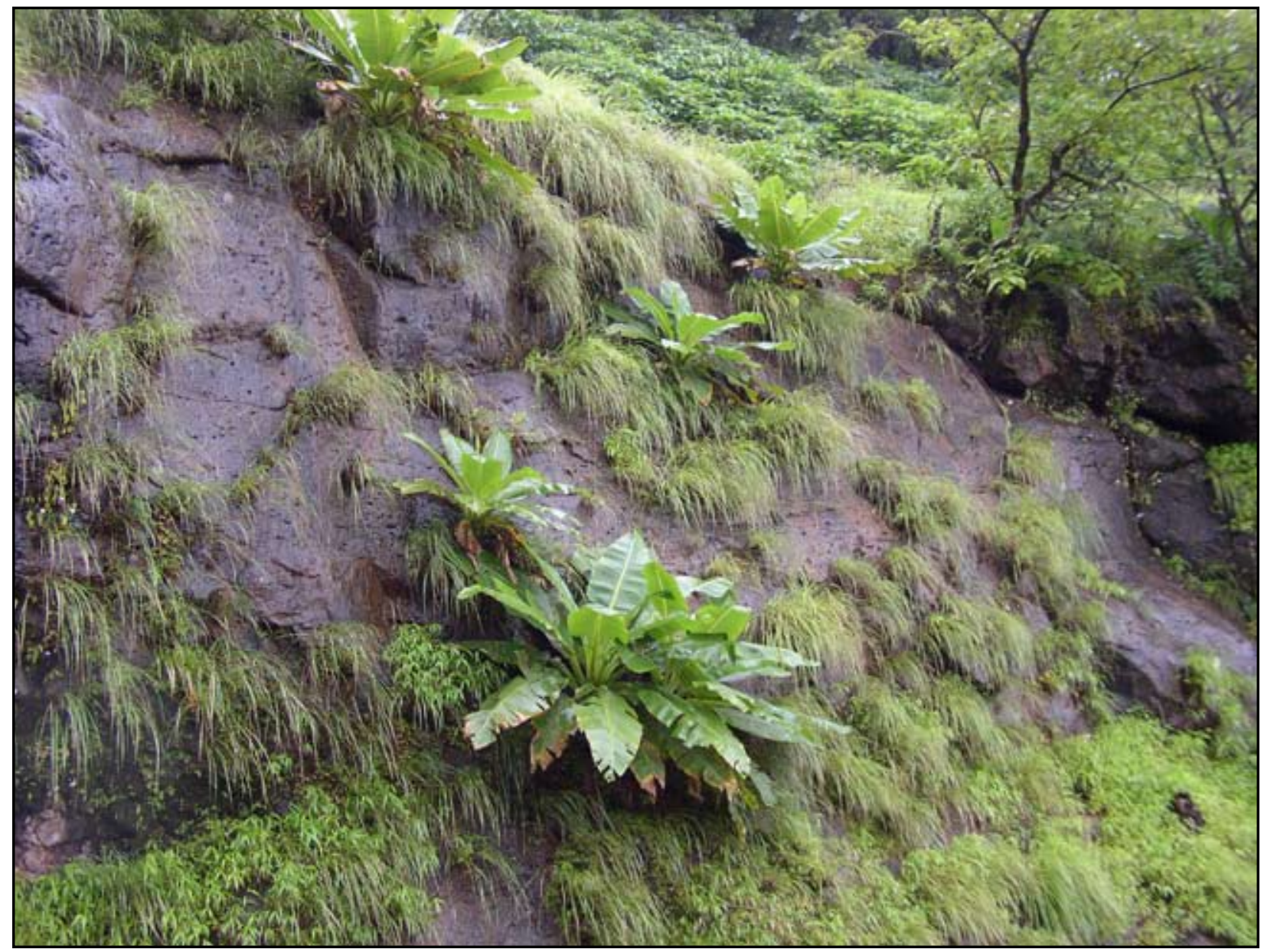

Figure 2. Wild Ensete superbum growing in the Sayhadri Hills, Western Ghats, Maharashtra, India (Photo: D.Q. Fuller, Sept. 2003). 
the wetter hill ranges of the Andhra Pradesh (Ellis 1987, Fischer 1928, Matthew 1995). In Western India, the Himalayas and Ganges plains, there are no wild Musaceae (cf. Duthie 1903, Hooker 1872-1897, Shetty \& Singh 1991), and suitable habitats are sporadic through central India and the eastern peninsular hill ranges. In the hills of Orissa, wild $M$. balbisiana favors rocky cliffs (Figure 3 ), while Musa ornata Roxb. (syn. M. rosacea Jacq.) grows in deep mud along sluggish streams in lower-lying valleys (Haines 1921-1925, cf. Singh et al. 2001). Musa ornata is also reported from the eastern Himalayan zone (Bengal and Sikkim) and it is noted that meristems (scapes) can be eaten after boiling or dried to make flour (Ambasta et al. 1986). Musa ornata occurs as far west as the Vindhyas in western Madhya Pradesh, but north of the Narmada (Dhar and Jhabua Districts; Singh et al. 2001), and as far south as the Rampa Hills of northeast Andhra, north of the Godavari River (Fischer 1928).

Under the wetter conditions that prevailed in the early Holocene and the terminal Pleistocene (Madella \& Fuller 2006, Prasad \& Enzel 2006), wild bananas must have been more widespread, in particular across the small hill ranges along the east side of the peninsula and through the central Indian hills. The disjunct distributions of $M$. balbisiana and $M$. acuminata in southern India and Sri Lanka presumably attest to migration and separation of populations from the northeast, and ultimately Southeast Asia through the processes of interglacial wetting and glacial drying. Nevertheless, the environments that seem to have contributed to Neolithic agricultural origins in India were not the kinds of environments where wild bananas grow. Namely, early agriculture was practiced in drier areas, such as the semi-arid savannah and deciduous woodland mosaics of the peninsula, or the seasonal wet river valleys and plains of the Gangetic region (see Fuller 2006a). Wild bananas were unlikely to have been part of the environments where food production began in South Asia.

\section{Bananas in South China}

Bananas are cultivated in the southern tropical areas of China, such as Yunnan, Guangxi and Guangdong, and

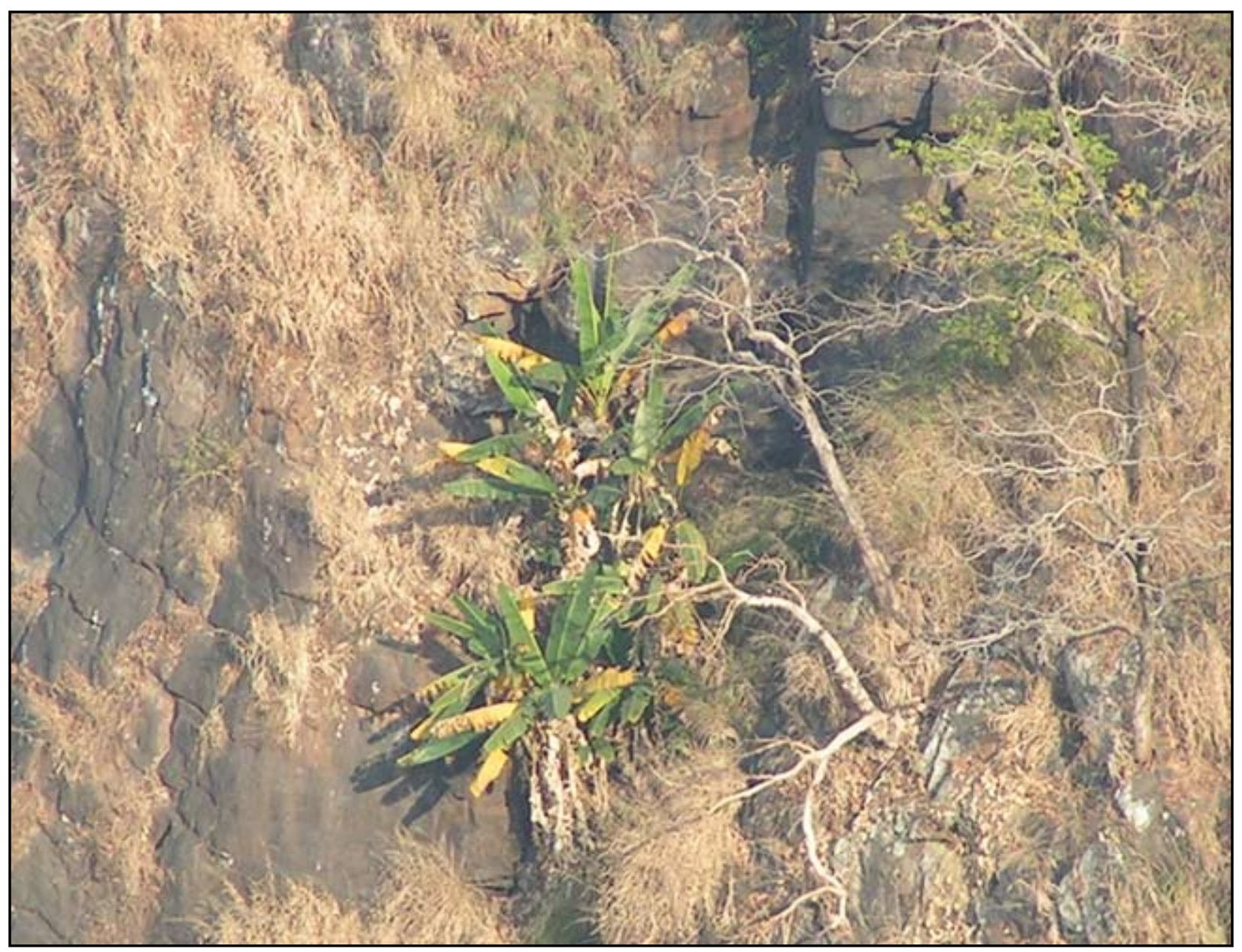

Figure 3. Wild Musa balbisiana, growing in the Simlipal Hills, Orissa, India (Photo: D.Q. Fuller, Dec. 2004). 


\section{Fuller \& Madella - Banana Cultivation in South Asia and East Asia: A review of the evidence from archaeology and linguistics}

eastwards to Fujian and Taiwan. Cultivars are mainly sweet AAA triploids, but BBB cultivars are also reported (Wu \& Kress 2000). As is true in South Asia, bananas are not usually staple foods. In order to track bananas archaeologically, one must contend with the problem that there are several wild Musaceae in south China (Wu \& Kress 2000), certainly more species than in India. This is especially true in the Yunnan region in the southwest. These include the small herbaceous Musella lasiocarpa (Franch.) C.Y. Wu, Ensete glaucum (Roxb.) Cheesman and Ensete wilsonii W.J. Tutcher, Musa coccinea H.C. Andrews, Musa itinerans Cheesman, Musa rubra Wall. ex Kurz, as well as the progenitor complex M. acuminata, and M. balbisiana. Many of these occur in the mountains above $1000 \mathrm{~m}$, although $M$. coccinea favours low elevations in ravines, which is also true of $M$. acuminata. Musa acuminata is re- portedly native in Yunnan and western Guangxi in low elevations and shady valleys. Almost all of these species of Musa, as well as Ensete and Musella, are reported to be cultivated, primarily for their leaves that are used for pig food (Wu \& Kress 2000). Other species have been recognized as ornamentals, such as Musa sanguinea Hook.f., a native of Assam, or the hardy bananas Musa basjoo Siebold \& Zucc., probably introduced to Japan and Korea; the wild status of the latter on Taiwan and Ryukyu Islands remains problematic. Chiu et al. (2007) accept only the endemic Musa formosana (Warb. ex Schum.) B. Hayata as native, and suggest that $M$. balbisiana in Taiwan is a recently naturalized introduction; this implies that $M$. basjoo is merely a cultivar that might occasionally be feral (i.e., naturalized). The approximate northern limit of wild Musaceae in China is indicated in Figure 4.

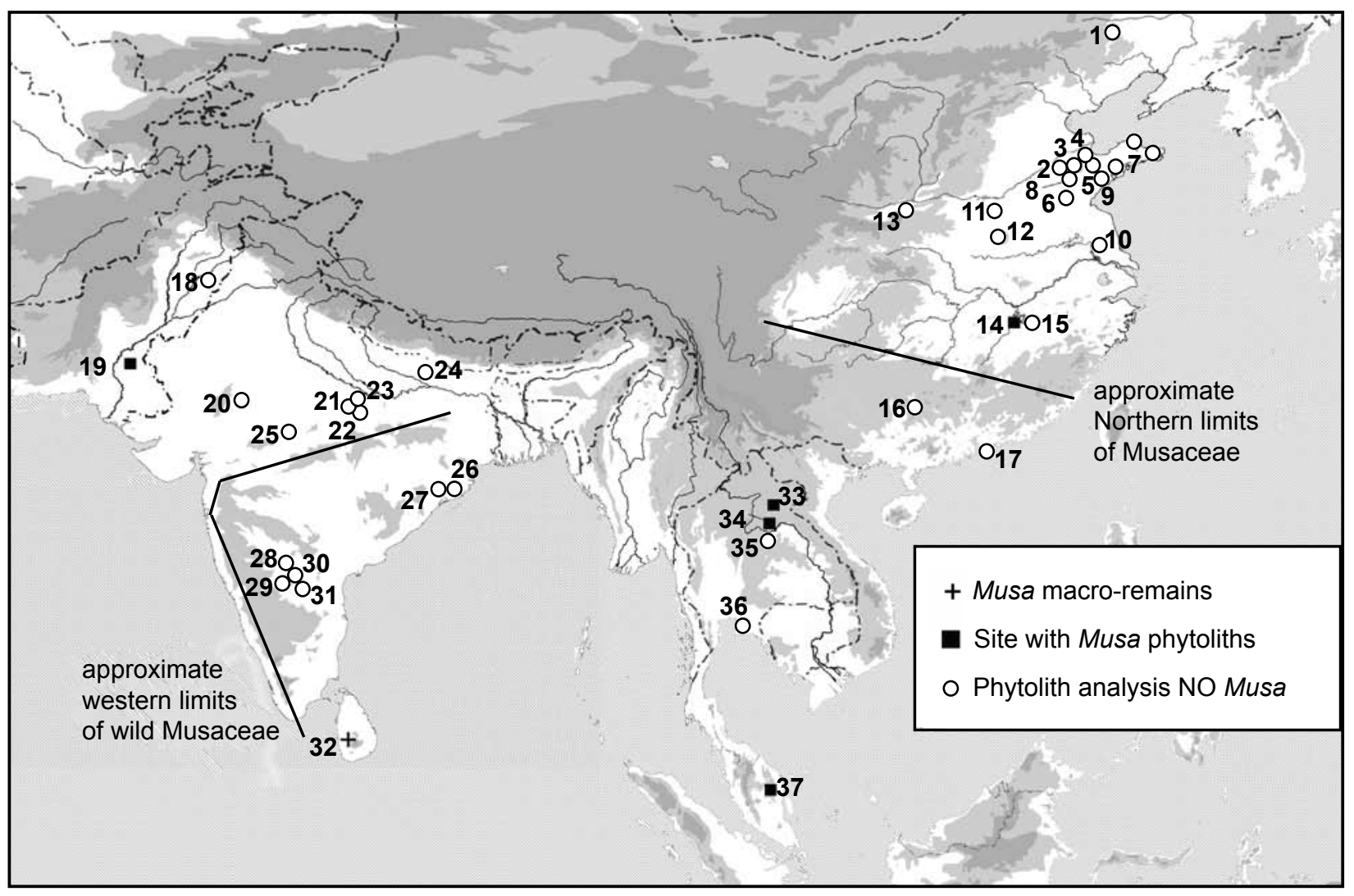

Figure 4. Map of archaeobotanical analyses (mainly phytoliths) discussed in this paper; references for South Asian and Chinese sites in Tables 1 and 2. Also indicated are approximate northern and western limits of wild Musaceae. Sites: 1. Shangjifangyingzi, 2. several sites: Wangfu, Yuezhang, Mengzhuang, Pingyinzhanggou, Pengzia; 3. Xihe and Chengziya, 4. Dinggong, 5. several sites: Qianbuxia, Lujiakou, Sihzixing, Lijiabu, Anqingudui; 6. several sites: Xigongqiao, Zhuangxili, Lianggudui, Gaogudi, Xigudui, Shengzhongli; 7. Shandong peninsula shell middens (several); 8. Dongjiaying and Qiaojiatun; 9. several sites: Yingjiacheng, Changgouzhangshanwa and Yuhuangding; 10. Longqiuzhuang, 11. Ying Valley (several sites), 12. Yangzhuang/ Zhumadian, 13. ; Quanhu 14. Poyang Lake, 15. Diaotonghuan, 16. Zengpiyan; 17. Sha Ha, Sai King, Hong Kong; 18. Harappa, 19. Kot Diji, 20. Balathal, 21. Mahagara, 22. Koldihwa, 23. Chopanimoando, 24. Lahuradewa Lake, 25. Nagauri \& Davrajpur, 26. Golbai Sassan, 27. Gopalpur, 28. Budihal, 29. Sanganakallu, 30. Kupgal, 31. Palavoy, 32. Beli-Lena (Musa macro-remains); 33 Plain of Jars/Ban Ang; 34. Lao Pako 35. Lake Kumphawapi 36. Khok Phanom Di; 37. Gua Chawas. 
Under the warmer and wetter conditions of the early Holocene (e.g., Dykoski et al. 2005, Madella \& Fuller 2006, Ren \& Beug 2002) these species would have occurred farther north, at least through the middle Yangtze basin. Judging by the modern distribution of wild rice and where it probably occurred in the past, we can expect wild bananas to have occurred further north. Rice has been found archaeologically, and perhaps still wild into the Huai River and Shandong (Fuller et al. 2007, 2008). In addition, historical sources from Tang Dynasty attest to wild rice into Shandong and broadly the lower Yellow river region in the mid Holocene (Ho 1977, You 1987). Such places are considerably north of the modern limits of wild rice, which requires sub-tropical or tropical wetlands free of substantial frosts. We can expect bananas to have also extended their range further north, but not quite as far as rice. In particular we should take into account those species that occur at higher elevation (and are thus adapted to cooler conditions), as these might more readily extend northwards but at progressively lower elevations. In this group we should probably consider Musella, E. glaucum, M. rubra and $M$. balbisiana as all possible producers of banana type phytoliths in the Poyang Lake area (see Figure 4). Evidence from the phytolith sequence there suggests that Musaceae (presumably wild) were present around 14,000-12,000 BP, and were continuously present from c. 4500 BP then disappear from the record in the Middle Yangzte region within the last 1000 years (Zhao \& Piperno 2000).
1989), is important as it attests to traditions of utilisation of indigenous wild $M$. balbisiana and possibly $M$. acuminata. However, little more can be said about other potential early use of these species in South Asia.

While phytoliths provide a firmer basis for tracking cultivated bananas (Mbida et al. 2000, 2006, Wilson 1985), phytolith analyses in South Asia are still few and far between. In Table 1, we have collected studies of phytoliths from South Asia (Figure 4). As can be seen, despite data from 16 sites, and evidence for crops such as wheat/barley or rice from many, Musa type phytoliths (i.e., volcaniform; Ball et al. 2006) have only been reported from one site, Kot Diji. Although studies are still limited in both South and East Asia, evidence for Musaceae phytoliths is limited to one site each in South Asia and China. Only in the case of Kot Diji, Pakistan, were these phytoliths found on an archaeological site, implying human use.

Cultivation of sweet banana hybrids on an intensive scale in Sindh began only in the mid 20th century A.D. Nevertheless Harappan period phytolith evidence from Kot Diji implies some cultivation by the 20th century B.C., or even earlier (Fuller \& Madella 2001:342, Madella 2003). This is suggested by clear Musaceae (volcaniform) phytoliths (Figure 5) collected from the stratigraphic section at Kot Diji, associated with Mature Harappan material culture, putting this at 1900-2000 B.C. at the latest (Madella

\section{The Archaeobotany of South Asia and the Limited Evidence of Bananas}

Archaeobotany has a long tradition in South Asia and has been producing data at an increasing rate in recent years (Fuller 2008:193). Data from macro-remains is now available from more than 120 sites, and there is a reasonable basis from which to infer the broad patterns of early cerealpulse agriculture in most parts of the subcontinent (Fuller 2006a). Nevertheless, this contributes little to our understanding of vegeculture crops, including bananas, and some tubers that may indeed have been important in the earlier phases of agriculture in some regions (Fuller 2006b:193-194). The one site with macro-remains evidence for wild bananas, an early Holocene hunter-gatherer cave site in Sri Lanka (Kajale

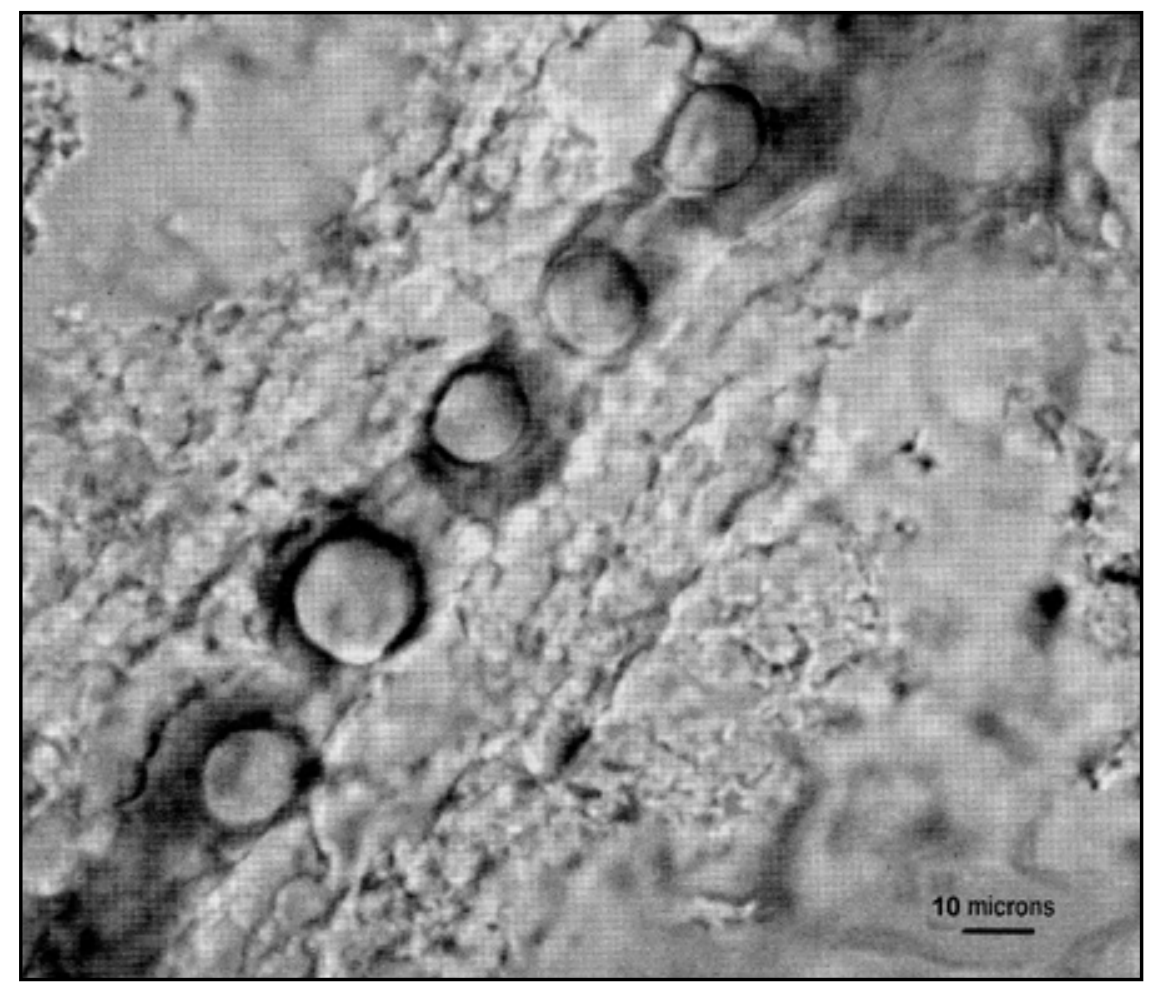

Figure 5. Musa phytoliths from Harappan period Kor Diji, Sindh, Pakistan (from Madella 2003). 


\section{Fuller \& Madella - Banana Cultivation in South Asia and East Asia: A review of the evidence from archaeology and linguistics}

Table 1. Sites with phytolith analyses in South Asia: Selected morphtypes of probable economic species.

\begin{tabular}{|c|c|c|c|c|c|c|c|c|}
\hline Site (source) & Culture \& Date & 쫑 & $\begin{array}{l}\text { OO } \\
\text { N̦ } \\
\text { o. } \\
\text { ․ }\end{array}$ & 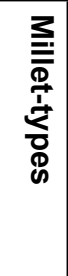 & 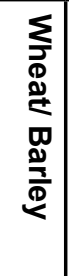 & 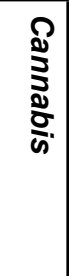 & 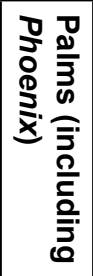 & $\begin{array}{l}\text { Agriculture inferred from macro- } \\
\text { remains from site/culture }\end{array}$ \\
\hline $\begin{array}{l}\text { Kot Diji, Sindh } \\
\text { (Madella 2003) }\end{array}$ & $\begin{array}{l}\text { Early Harappan } \\
\text { 3000-2500 B.C. }\end{array}$ & 0 & 0 & 0 & $\mathrm{X}$ & 0 & $\mathrm{X}$ & $\begin{array}{l}\text { Wheat \& Barley agriculture in } \\
\text { region (Fuller \& Madella 2001) }\end{array}$ \\
\hline $\begin{array}{l}\text { Kot Diji, Sindh } \\
\text { (Madella 2003) }\end{array}$ & $\begin{array}{l}\text { Mature Harappan } \\
\text { 2500-1900 B.C. }\end{array}$ & $\mathrm{X}$ & 0 & $\mathrm{X}$ & $x$ & 0 & $\mathrm{X}$ & Same as above \\
\hline $\begin{array}{l}\text { Harappa, Punjab } \\
\text { (Madella 2003) }\end{array}$ & $\begin{array}{l}\text { Early Harappan } \\
\text { 3000-2500 B.C. }\end{array}$ & 0 & 0 & $\mathrm{X}$ & $\mathrm{X}$ & 0 & 0 & $\begin{array}{l}\text { Wheat \& barley, Panicum } \\
\text { sumatrense, pulses, other } \\
\text { millets (Weber 1999, 2003) }\end{array}$ \\
\hline $\begin{array}{l}\text { Harappa, Punjab } \\
\text { (Madella 2003) }\end{array}$ & $\begin{array}{l}\text { Mature Harappan } \\
\text { 2500-1900 B.C. }\end{array}$ & 0 & 0 & $\mathrm{X}$ & $\mathrm{X}$ & 0 & 0 & Same as above \\
\hline $\begin{array}{l}\text { Harappa, Punjab } \\
\text { (Madella 2003) }\end{array}$ & $\begin{array}{l}\text { Late Harappan } \\
\text { 1900-1600 B.C. }\end{array}$ & 0 & $\mathrm{X}$ & $\mathrm{X}$ & $\mathrm{X}$ & $\mathrm{X}$ & 0 & Same as above with addition of rice. \\
\hline $\begin{array}{l}\text { Balathal, } \\
\text { Rajasthan } \\
\text { (Kajale \& } \\
\text { Eksambekar } \\
\text { 2001) }\end{array}$ & $\begin{array}{l}\text { Chalcolithic } \\
\text { 3000-2000 B.C. }\end{array}$ & 0 & $\mathrm{X}$ & $?$ & $?$ & 0 & 0 & Wheat \& barley, pulses (Kajale 1996) \\
\hline $\begin{array}{l}\text { Balathal, } \\
\text { Rajasthan } \\
\text { (Eksambekar \& } \\
\text { Kajale 2007) }\end{array}$ & $\begin{array}{l}\text { Early Historic } \\
200 \text { B.C.- A.D. } 200\end{array}$ & 0 & $x$ & $\mathrm{X}$ & $?$ & 0 & 0 & $\begin{array}{l}\text { Wheat, barley, rice, millets, } \\
\text { pulses (Kajale 1996) }\end{array}$ \\
\hline $\begin{array}{l}\text { Sanganakallu, } \\
\text { Karnataka } \\
\text { (Madella et al. } \\
\text { n.d.a, Weisskopf } \\
\text { 2005) }\end{array}$ & $\begin{array}{l}\text { Ashmound } \\
\text { Tradition } \\
\text { 2000-1400 B.C. }\end{array}$ & 0 & 0 & $x$ & 0 & 0 & $X$ & $\begin{array}{l}\text { Brachiaria ramosa (L.) Stapf, } \\
\text { pulses, some wheat \& barley } \\
\text { (Fuller et al. 2004) }\end{array}$ \\
\hline $\begin{array}{l}\text { Budihal, } \\
\text { Karnataka } \\
\text { (Kajale \& } \\
\text { Eksambekar } \\
\text { 1997) }\end{array}$ & $\begin{array}{l}\text { Ashmound } \\
\text { Tradition } \\
\text { 2300-1700 B.C. }\end{array}$ & 0 & 0 & $x$ & 0 & 0 & 0 & $\begin{array}{l}\text { Brachiaria ramosa, pulses, } \\
\text { some wheat \& barley (cf. } \\
\text { Kajale \& Eksambekar 1997) }\end{array}$ \\
\hline $\begin{array}{l}\text { Palavoy, } \\
\text { Andhra Pradesh } \\
\text { (Madella et al. } \\
\text { n.d.b) }\end{array}$ & $\begin{array}{l}\text { Ashmound } \\
\text { Tradition } \\
\text { 1910-1700 B.C. }\end{array}$ & $?$ & $?$ & $x$ & $?$ & $?$ & $?$ & $\begin{array}{l}\text { Expected as above } \\
\text { (Fuller et al. 2004) }\end{array}$ \\
\hline $\begin{array}{l}\text { Kupgal, } \\
\text { Karnataka } \\
\text { (Madella et al. } \\
\text { n.d.b) }\end{array}$ & $\begin{array}{l}\text { Ashmound } \\
\text { Tradition } \\
\text { (2000 -1500 B.C.?) }\end{array}$ & 0 & 0 & $X$ & 0 & 0 & 0 & $\begin{array}{l}\text { Expected as above } \\
\text { (Fuller et al. 2004) }\end{array}$ \\
\hline $\begin{array}{l}\text { Lahuradewa } \\
\text { Lake core, } \\
\text { Uttar Pradesh } \\
\text { (Saxena et } \\
\text { al. 2006) }\end{array}$ & $\begin{array}{l}\text { Non- } \\
\text { archaeological, } \\
\text { Holocene } \\
\text { sequence }\end{array}$ & 0 & $x$ & $?$ & 0 & 0 & 0 & $\begin{array}{l}\text { Rice, small millet (?), wheat \& } \\
\text { barley after } 2500 \text { B.C. (Saraswat } \\
\text { 2005, see Fuller 2006b:42) }\end{array}$ \\
\hline $\begin{array}{l}\text { Mahagara, } \\
\text { Uttar Pradesh } \\
\text { (Harvey 2006) }\end{array}$ & $\begin{array}{l}\text { Late Neolithic } \\
\text { 1800-1600 B.C. }\end{array}$ & 0 & $X$ & $X$ & 0 & 0 & $x$ & $\begin{array}{l}\text { Rice, millet, pulses, wheat \& } \\
\text { barley (Harvey } 2006, \text { Harvey \& } \\
\text { Fuller 2005, Harvey et al. } 2005 \text { ) }\end{array}$ \\
\hline
\end{tabular}




\begin{tabular}{|c|c|c|c|c|c|c|c|c|}
\hline Site (source) & Culture \& Date & 쫑 & $\begin{array}{l}\text { 옹 } \\
\text { N. } \\
\text { 은 }\end{array}$ & 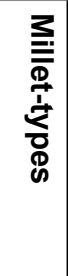 & 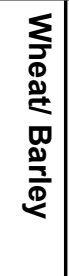 & 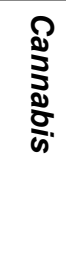 & 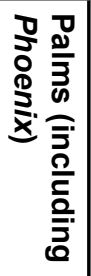 & $\begin{array}{l}\text { Agriculture inferred from macro- } \\
\text { remains from site/culture }\end{array}$ \\
\hline $\begin{array}{l}\text { Koldihwa, } \\
\text { Uttar Pradesh } \\
\text { (Harvey 2006) }\end{array}$ & $\begin{array}{l}\text { Late Neolithic } \\
\text { \& Iron Age } \\
1800-500 \text { B.C. }\end{array}$ & 0 & $x$ & $x$ & 0 & 0 & $X$ & Same as above \\
\hline $\begin{array}{l}\text { Chopanimando } \\
\text { (Harvey 2006) }\end{array}$ & $\begin{array}{l}\text { Mesolithic ? } \\
10000-3000 \text { B.C. }\end{array}$ & 0 & 0 & 0 & 0 & 0 & $\mathrm{X}$ & $\begin{array}{l}\text { Unclear. Foragers with wild rice } \\
\text { (impression) (Sharma et al. 1980) }\end{array}$ \\
\hline $\begin{array}{l}\text { Golbai Sassan, } \\
\text { Orissa (Harvey } \\
2006 \text { ) }\end{array}$ & 1400-1200 B.C. & 0 & $x$ & $x$ & 0 & 0 & $X$ & $\begin{array}{l}\text { Rice, pulses, small millets } \\
\text { (Harvey et al. 2006) }\end{array}$ \\
\hline $\begin{array}{l}\text { Gopalpur, Orissa } \\
\text { (Harvey 2006) }\end{array}$ & 1400-1200 B.C. & 0 & $\mathrm{X}$ & $\mathrm{X}$ & 0 & 0 & $x$ & Same as above \\
\hline $\begin{array}{l}\text { Bajpur, Orissa } \\
\text { (Harvey 2006) }\end{array}$ & $\begin{array}{l}? \\
2000-1000 \text { B.C. }\end{array}$ & 0 & $x$ & 0 & 0 & 0 & $x$ & $\begin{array}{l}\text { Shifting cultivators (?), no preserved } \\
\text { macro-remains (Harvey et al. 2006) }\end{array}$ \\
\hline $\begin{array}{l}\text { Malakhoja, } \\
\text { Orissa (Harvey } \\
2006)\end{array}$ & $\begin{array}{l}? \\
2000-1000 \text { B.C. }\end{array}$ & 0 & 0 & 0 & 0 & 0 & $x$ & Same as above \\
\hline $\begin{array}{l}\text { Nagauri \& } \\
\text { Davrajpur } \\
\text { reservoirs (Shaw } \\
\text { et al. 2007:175) }\end{array}$ & 300 B.C.-500 A.D. & 0 & 0 & 0 & 0 & $0^{*}$ & 0 & Irrigated rice, *Cannabis type pollen \\
\hline
\end{tabular}

2003). Given the distribution of wild Musaceae in South Asia, and the climate at that time (Asouti \& Fuller 2008, Madella \& Fuller 2006), it is unlikely that these could derive from the ancient presence of wild Musa or Ensete. The possibility that a species was cultivated as a garden ornamental or as a source of fiber and raw materials (e.g., for paper) cannot be ruled out. Indeed, one of these nonculinary uses of Musa/Ensete might be a more plausible explanation for these phytoliths than an early dispersal of edible cultivated bananas from Island Southeast Asia by the third millennium B.C. However, given the absence of a clear scribal tradition in the Indus Valley (Farmer et al. 2004), the paper hypothesis should be viewed with caution; but other fiber uses are plausible. In earlier studies, the presence of banana cultivation had already been suggested on the basis of a rare painted motif on pottery, present apparently only at Mohenjodaro (Sindh, Pakistan). It was first identified as possible banana plants by MacKay (1938:220), and subsequently by Wheeler (1968:85). The incomplete representations are by no means definitive and more recent commentators have taken no clear position on these (e.g., Fuller \& Madella 2001, McKean 1983:32), but a selection of these possible banana motifs is reproduced here (Figure 6).

The phytoliths observed in Kot Diji were few and still in anatomical connection (silica skeletons) and it was not possible to acquire all morphometries. However, the best- preserved group was photographed (see Figure 5) and crater width values measured. These varied between 11.4 $\mu \mathrm{m}$ and $15.7 \mu \mathrm{m}$. Only one base length was recorded with a value of $34.2 \mu \mathrm{m}$. Notwithstanding the fact that volcaniform phytoliths of $M$. balbisiana and $M$. acuminata cannot be distinguished by morphotype analysis, the few crater measurements are all above the maximum crater width of both $M$. balbisiana and $M$. acuminata possibly suggesting that hybridization had already occurred. The aridity of this region is such that pure $M$. acuminata varieties would be unlikely to survive. For more semi-arid areas, like much of India and large parts of Africa, hybrids between M. acuminata and $M$. balbisiana are cultivated and these appear to be better adapted to less water (Simmonds 1962). This leads us to suggest that hybridization is likely to have already occurred before Musa spread into Sindh. This raises the question of where hybridization between $M$. acuminata and $M$. balbisiana might have occurred. Is Sri Lanka a possible area for hybridization with cultivated $M$. acuminata bananas? If so, how did cultivated acuminata bananas get there? Hybridization here, and/or in the New Guinea area now seems more plausible than hybridization in northern Southeast Asia (from Burma through eastern India) as Simmonds (1962, 1995) had hypothesized, given the inferred later history and minor role of bananas in those areas (see historical linguistics evidence below). This hybridization by/during the later third millennium time horizon is significant in terms of preparing bananas for 


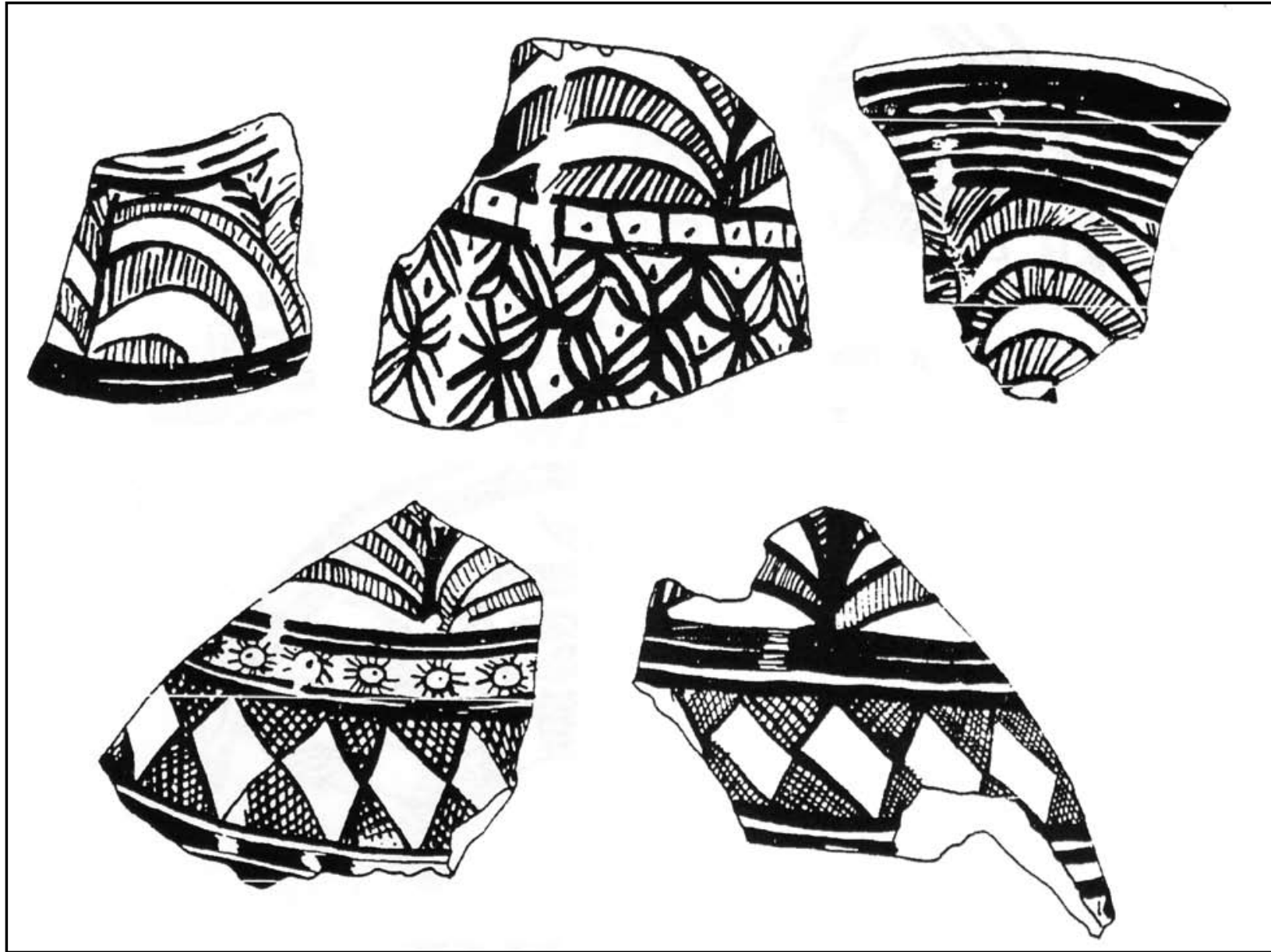

Figure 6. Examples of the putative Musaceae motifs from painted pottery at Mohenjodaro, Sindh, Pakistan (after Marshall 1931).

cultivation in Africa and implying an early dispersal across the Bay of Bengal/eastern Indian Ocean.

\section{The Archaeobotany of China and the Limited Evidence of Bananas}

Archaeobotany in China has made major quantitative progress in recent years, as programs of systematic flotation have become more widespread (Zhao 2008). Nevertheless, systematic sampling has been heavily biased towards central and northern China, with a research focus on the origins of civilization and early millet agriculture (e.g., Fuller \& Zhang 2007, Lee et al. 2007, Zhao 2008). This has been the part of China with the most phytolith analyses, especially in Shandong (see Table 2, Figure 4). Additionally, there has been increasing work on early rice farming, especially in the Lower Yangtze region (Fuller et al. 2007, 2009, Zheng et al. 2007). Work in the more tropical south is still much more limited (cf. Lu 2006); full phytolith analyses are available from some cave sites (Zhao 1998, 2003), and these have not yet produced Musa type phytoliths. In many cases phytolith analyses have focused on rapid processing methods to retrieve phytoliths for the purpose of identifying rice paddy fields or for measuring rice bulliforms (e.g., Jin et al. 2007, Zheng et al. 2004, Zou et al. 2000). As a result, such analyses are unlikely to pick up Musa even if present. It remains the case that the only site with presence of Musa type phytoliths (volcaniform) is that of Poyang Lake, in which wild Musaceae, as part of a more tropical flora, were present in the area from the mid to late Holocene (Zhao \& Piperno 2000).

Further south in mainland Southeast Asia, phytolith analyses have been even fewer. Musa phytoliths were recovered from two Iron Age sites in Laos, Xiang Khoang at Ban Ang ('plain of jars') and Lao Pako (Bowdery 1999), and probably date to 0-500 A.D. Lake sediments from Kumphawapi in northern Thailand lacked Musa types, although rice was present (Bowdery 1999). Another study of phytoliths from valley sediments north of the site of Khok Phanom Di failed to turn up Musa morphotypes, but other potential tree cultivars such as Cocos, Areca, Nypa and Borassus palms were tentatively identified and rice types were present (Kealhofer \& Piperno 1996). In Peninsular Malaysia a sediment sample from the site of Gua Chawas yielded Musa phytoliths from Hoabhinian (perhaps 5000 
Table 2. Sites with phytolith analyses in China: Selected morphotypes of probable economic species.

\begin{tabular}{|c|c|c|c|c|c|c|c|c|}
\hline Site (source) & Culture \& Date & కุ & 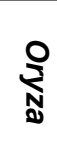 & ڤે. & 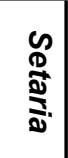 & 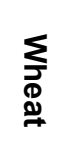 & $\begin{array}{l}\text { Agriculture } \\
\text { inferred from } \\
\text { macro-remains } \\
\text { from site/culture }\end{array}$ & Source \\
\hline Shangjifangyingzi & $\begin{array}{l}\text { Xiajiadian Culture } \\
\text { (both lower and upper } \\
\text { layers) } 2300-300 \text { B.C. }\end{array}$ & 0 & 0 & 0 & 0 & 0 & Millet & Wang et al. 2007 \\
\hline Wangfu & $\begin{array}{l}\text { Spring-Autumn period } \\
770-476 \text { B.C. }\end{array}$ & 0 & 0 & 0 & 0 & 0 & Millet & $\operatorname{Jin} 2006$ \\
\hline Yuezhuang & $\begin{array}{l}\text { Houli Culture } \\
\text { (6000-5000 B.C.) }\end{array}$ & 0 & 0 & 0 & 0 & 0 & Millet(?) \& Rice(?) & $\operatorname{Jin} 2008$ \\
\hline Xihe & $\begin{array}{l}\text { Houli Culture } \\
\text { (6000-5000 B.C.) }\end{array}$ & 0 & 0 & $?$ & $?$ & 0 & Millet(?) \& Rice(?) & Jin 2006 \\
\hline Pengzia & $\begin{array}{l}\text { Houli Culture } \\
(6000-5000 \text { B.C.) }\end{array}$ & 0 & 0 & 0 & 0 & 0 & Millet(?) \& Rice(?) & $\operatorname{Jin} 2008$ \\
\hline Yuhuangding & $\begin{array}{l}\text { Late Beixin - Early } \\
\text { Dawenkou } \\
\text { (4000-3000 B.C.) }\end{array}$ & 0 & 0 & $x$ & $x$ & 0 & Millets & $\operatorname{Jin} 2008$ \\
\hline Qiaojiatun & $\begin{array}{l}\text { Mid Dawenkou } \\
\text { (3000-2600 B.C.) }\end{array}$ & 0 & 0 & 0 & 0 & 0 & Millets & $\operatorname{Jin} 2008$ \\
\hline Dongjiaying & $\begin{array}{l}\text { Late Dawenkou } \\
\text { (2700-2300 B.C.) }\end{array}$ & 0 & 0 & 0 & 0 & 0 & Millets & $\operatorname{Jin} 2008$ \\
\hline Yingjiacheng & $\begin{array}{l}\text { Longshan } \\
(2300-1900 \text { B.C.) }\end{array}$ & 0 & 0 & $x$ & $x$ & 0 & Millets and rice & Jin 2008 \\
\hline $\begin{array}{l}\text { Changgouzhang- } \\
\text { shanya }\end{array}$ & $\begin{array}{l}\text { Longshan } \\
(2300-1900 \text { B.C.) }\end{array}$ & 0 & 0 & 0 & 0 & 0 & Millets and rice & $\operatorname{Jin} 2008$ \\
\hline Zhuanglixi & $\begin{array}{l}\text { Longshan } \\
(2300-1900 \text { B.C.) }\end{array}$ & 0 & $x$ & $x$ & $x$ & 0 & Millets and rice & Jin 2008 \\
\hline Lianggudui & $\begin{array}{l}\text { Longshan } \\
\text { (2300-1900 B.C.) }\end{array}$ & 0 & 0 & 0 & 0 & 0 & Millets and rice & $\operatorname{Jin} 2008$ \\
\hline Gaogudui & $\begin{array}{l}\text { Longshan } \\
(2300-1900 \text { B.C. })\end{array}$ & 0 & 0 & $x$ & $x$ & 0 & Millets and rice & Jin 2008 \\
\hline Xigudui & $\begin{array}{l}\text { Longshan } \\
(2300-1900 \text { B.C.) }\end{array}$ & 0 & 0 & 0 & 0 & 0 & Millets and rice & $\operatorname{Jin} 2008$ \\
\hline Shengzhongji & $\begin{array}{l}\text { Longshan } \\
(2300-1900 \text { B.C.) }\end{array}$ & 0 & 0 & 0 & 0 & 0 & Millets and rice & $\operatorname{Jin} 2008$ \\
\hline Tagangsi & $\begin{array}{l}\text { Longshan } \\
(2300-1900 \text { B.C.) }\end{array}$ & 0 & $x$ & 0 & 0 & 0 & Millets and rice & Jin 2008 \\
\hline Anqiugudui & $\begin{array}{l}\text { Longshan Culture } \\
(2300-1900 \text { B.C. })\end{array}$ & 0 & 0 & $?$ & $?$ & 0 & Rice and millet & $\operatorname{Jin} 2008$ \\
\hline Guangudui & $\begin{array}{l}\text { Longshan Culture } \\
\text { (2300-1900 B.C.) }\end{array}$ & 0 & 0 & 0 & 0 & 0 & Rice and millet & $\operatorname{Jin} 2008$ \\
\hline Lujiakou & $\begin{array}{l}\text { Longshan Culture } \\
(2300-1900 \text { B.C.) }\end{array}$ & 0 & $X$ & $x$ & $X$ & 0 & Rice and millet & $\operatorname{Jin} 2008$ \\
\hline Shizihang & $\begin{array}{l}\text { Longshan Culture } \\
\text { (2300-1900 B.C.) }\end{array}$ & 0 & $x$ & $x$ & $x$ & 0 & Rice and millet & $\operatorname{Jin} 2008$ \\
\hline Lijiabu & $\begin{array}{l}\text { Longshan- Yueshi } \\
\text { (2300-1400 B.C.) }\end{array}$ & 0 & $x$ & $x$ & $x$ & 0 & Rice and millet & Jin 2008 \\
\hline Chengziya & Yueshi (1900-1400 B.C.) & 0 & $\mathrm{X}$ & $\mathrm{X}$ & $X$ & 0 & Rice and millet & Jin 2008 \\
\hline
\end{tabular}


Fuller \& Madella - Banana Cultivation in South Asia and East Asia: A review of the evidence from archaeology and linguistics

\begin{tabular}{|c|c|c|c|c|c|c|c|c|}
\hline Site (source) & Culture \& Date & కై & 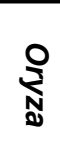 & 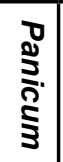 & 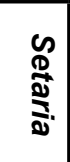 & 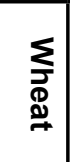 & \begin{tabular}{|l} 
Agriculture \\
inferred from \\
macro-remains \\
from site/culture
\end{tabular} & Source \\
\hline Mengzhuang & $\begin{array}{l}\text { Spring \& Autumn } \\
\text { (771-476 B.C.) }\end{array}$ & 0 & $x$ & $\mathrm{x}$ & $x$ & 0 & Rice(?) and millet & $\operatorname{Jin} 2008$ \\
\hline Pingyingzhangzou & $\begin{array}{l}\text { Spring \& Autumn } \\
\text { (771-476 B.C.) }\end{array}$ & 0 & 0 & $x$ & $x$ & $x$ & Rice(?) and millet & $\operatorname{Jin} 2008$ \\
\hline Dinggong & $\begin{array}{l}\text { Longshan Culture } \\
(2300-1900 \text { B.C.) }\end{array}$ & 0 & 0 & 0 & 0 & 0 & Rice and millet & $\operatorname{Jin} 2006$ \\
\hline Qianbuxia & $\begin{array}{l}\text { Dawenkou Culture } \\
\text { (4000-2300 B.C.) }\end{array}$ & 0 & 0 & $?$ & $?$ & 0 & Millet & $\operatorname{Jin} 2006$ \\
\hline Xigongqiao & $\begin{array}{l}\text { Dawenkou Culture(4000- } \\
\text { 2300BC) }\end{array}$ & 0 & 0 & 0 & 0 & 0 & Millet & $\operatorname{Jin} 2006$ \\
\hline $\begin{array}{l}\text { Shandong } \\
\text { peninsula shell } \\
\text { middens (several) }\end{array}$ & $\begin{array}{l}\text { Around } 3000 \text { B.C., } \\
\text { up to } 2800 \text { B.C. }\end{array}$ & 0 & $?$ & 0 & 0 & 0 & $?$ & $\operatorname{Jin} 2006$ \\
\hline $\begin{array}{l}\begin{array}{l}\text { Ying Valley } \\
\text { (several sites) }\end{array} \\
\end{array}$ & \begin{tabular}{|l|} 
Yangshao, Longshan \\
Cultures(5000-1800 B.C.) \\
\end{tabular} & 0 & $x$ & $x$ & $X$ & 0 & Millet and Rice & $\operatorname{Jin} 2007$ \\
\hline $\begin{array}{l}\text { Yangzhuang/ } \\
\text { Zhumadian }\end{array}$ & $\begin{array}{l}\text { Longshan, Erlitou } \\
\text { Cultures } \\
(2500-1500 \text { B.C.) }\end{array}$ & 0 & $x$ & 0 & 0 & 0 & Millet and rice & Qiang 1995 \\
\hline Longqiuzhuang & Neolithic site \& cemetary & 0 & $x$ & 0 & 0 & 0 & Rice & \begin{tabular}{|l} 
Huang \& \\
Zhang 2000
\end{tabular} \\
\hline Poyang Lake & Natural lake & $x$ & $x$ & 0 & 0 & 0 & $\begin{array}{l}\text { Rice cultivation } \\
\text { in region from } \\
\text { Early(?) Holocene }\end{array}$ & $\begin{array}{l}\text { Zhao \& Piperno } \\
2000\end{array}$ \\
\hline $\begin{array}{l}\text { Diaotonghuan } \\
\text { (Zhao 1998) }\end{array}$ & $\begin{array}{l}\text { Late Pleistocene to } \\
\text { Early Holocene cave }\end{array}$ & 0 & $x$ & 0 & 0 & 0 & $\begin{array}{l}\text { Rice cultivation } \\
\text { from Early(?) } \\
\text { Holocene }\end{array}$ & Zhao 1998 \\
\hline Zengpiyan & $\begin{array}{l}\text { 12000-8000BP (Late } \\
\text { Pleistocana to Early } \\
\text { Holocene cave) }\end{array}$ & 0 & 0 & 0 & 0 & 0 & Hunter-gathering & Zhao 2003 \\
\hline Quanhu & $\begin{array}{l}\text { Yangshao Culture } \\
\text { (5000-3000 B.C.) }\end{array}$ & 0 & $x$ & $\mathrm{x}$ & $x$ & 0 & Millet and Rice & Lu \& Zhang 2008 \\
\hline $\begin{array}{l}\text { Sha Ha, Sai King, } \\
\text { Hong Kong }\end{array}$ & $\begin{array}{l}\text { Late Neolithic-Bronze } \\
\text { Age (2000-1000 B.C.) }\end{array}$ & 0 & $x$ & 0 & 0 & 0 & Rice & Lu et al. 2005 \\
\hline
\end{tabular}

B.C.), Neolithic (after 2000 B.C.) and recent (Orang Asli) levels (Bowdery 1999). In Southeast Asian contexts such as these, the presence of wild Musaceae is highly likely and further archaeobotanical and paleoecological evidence is necessary before we can infer that these represented banana cultivation or consumption.

\section{Historical and Historical Linguistic Evidence}

In some of the earliest historical sources about South Asia, bananas get a mention. Thus Theophrastus' Enquiry into Plants (ca. 300 B.C.) refers to a tree "whose leaf is oblong in shape like the feathers of an Ostrich," recorded from
Alexander's eastern campaign, while later Pliny (c. A.D. 50 ) refers to its edible bunches of fruits (see Desmond 1992:2, 6). Within India, historical references date back to early Buddhist Pali texts, perhaps c. 400 B.C. (Achaya 1998). Such sources suggest that bananas were cultivated at that time in the alluvial plains of north India (e.g., the Ganges and presumably the upper Ravi and other Indus tributaries). In South India there are apparent references in some Old Tamil Sangam literature (c. 1800 B.P.?) (Achaya 1998).

In China the earliest historical sources are later, making reference to this southern fruit in the Late Han times, i.e., third century A.D. (Reynolds \& Fang 1940), but such sources are amongst the earliest to provide any details 


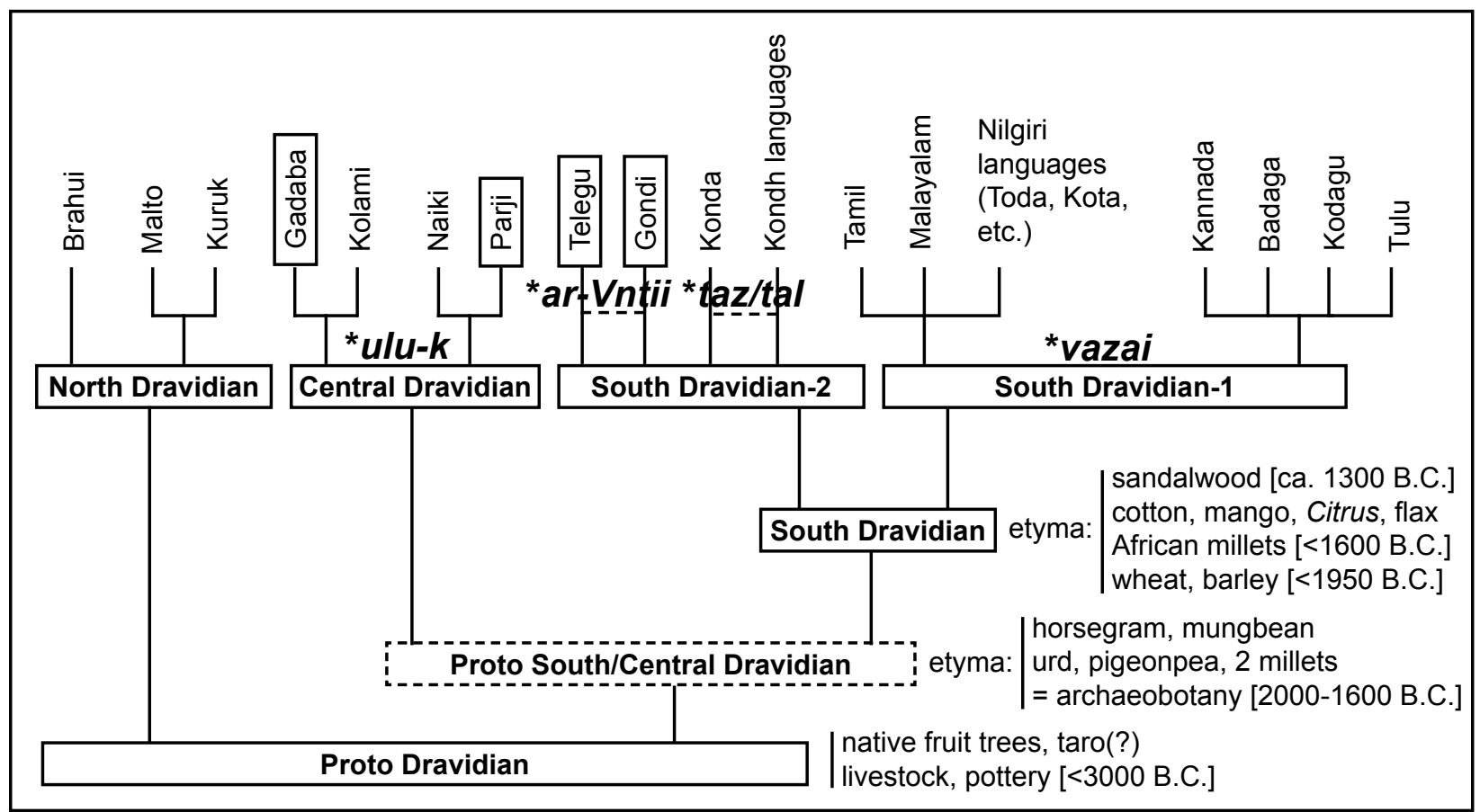

Figure 7. A representation of Dravidian Language family tree (based on Fuller 2007, Southworth 2006), with 4 banana proto-etyma ("ulu-k, *ar-Vntii, taz/tal, *vazai) indicated for sub-groups, and with selected etyma and archaeological dates indicated for earlier branches.

about southern Chinese plants, so these do not provide any clear indication of how much earlier bananas were grown in the region. Early Chinese sources make reference both to the sweet fruit and the production of fibers from the leaves and stems. A detailed description by Chi Han in his Description of Plants and Trees of the Southern Region (A.D. 304) indicates that bananas were grown in the Guangdong region at that time (Reynolds \& Fang 1940, Schafer 1967). Early Chinese terms included kanjiāo, pa-jiāo, compounded on the jiāo word, meaning a fibrous plant, or originally referring to straw or fuel (cf. Karlgren 1923:\#1065), much as the modern xiang-jiāo, in use since the 17th century, means 'sweet-jiāo' (Schafer 1967:168); today the preferred botanical name is simply 'big-jiao', da-jiao (Wu \& Kress 2000).

Historical linguistic inferences would similarly suggest that bananas were introduced to peninsular India in the order of 2000 years ago, after the separation of the main branches and sub-branches of the Dravidian language family (Figure 7). In recent years, we have been able to suggest a likely framework for constraining some of the dates of divergence between Dravidian language subfamilies based on the reconstructible etyma, especially of plants, and known archaeological dates for these species (Fuller 2003, 2006b, 2007, Southworth 2005, 2006). In Figure 7, the Dravidian family tree is shown with a selection of crop terms that can be constrained archaeologically to provide dates for earlier Proto-Languages, such as Proto-South Dravidian or Proto-South-Central Dravid- ian. On the upper part of the tree four reconstructed proto-form terms of bananas are indicated: these are Central Dravidian ( ${ }^{*}$ uluv/uluk), Telegu-Gondi ( ${ }^{*}$ ar-Vntti), South Dravidian(1) (*wāz-a-), and macro-Kondh ( ${ }^{*}$ taz/tal) (Southworth 2005:211, 227 n. 27). There are three implications from the data. First, that bananas were introduced long after the main subfamilies had divided, which suggests something in the past 2000 years or so. Second, it suggests different linguistic sources, i.e., different source loan words, hinting at multiple routes of introduction of the banana. This probably involved separate names associated with starchy and sweet varieties. Third, and significantly, banana terms appear much later than some other eastern introductions. For example both mangos and two kinds of Citrus fruits reconstruct to Proto-South Dravidian. Although these taxa plausibly originate in northeast India and spread overland from the north, they are present in some wood charcoal evidence from southern India from 1400-1300 B.C. (Asouti \& Fuller 2008). But sandalwood (Santalum album L.), Areca palms and coconuts (see Fuller 2007: Table 5), all of which more probably came to southern India from Indonesia/Malaysia (on Santalum, see Fischer 1938, Harbaugh \& Baldwin 2007; on Areca, see Zombrich 2007), also reconstruct to this period and Santalum has also been found in the wood charcoal record from deposits in this 1400-1300 B.C. horizon (Asouti \& Fuller 2008). This suggests that the Iron Age arrival of bananas to peninsular India lagged behind certain other introductions from the east. These other introductions from Southeast Asia, whether overland from the northeast 


\section{Fuller \& Madella - Banana Cultivation in South Asia and East Asia: A review of the evidence from archaeology and linguistics}

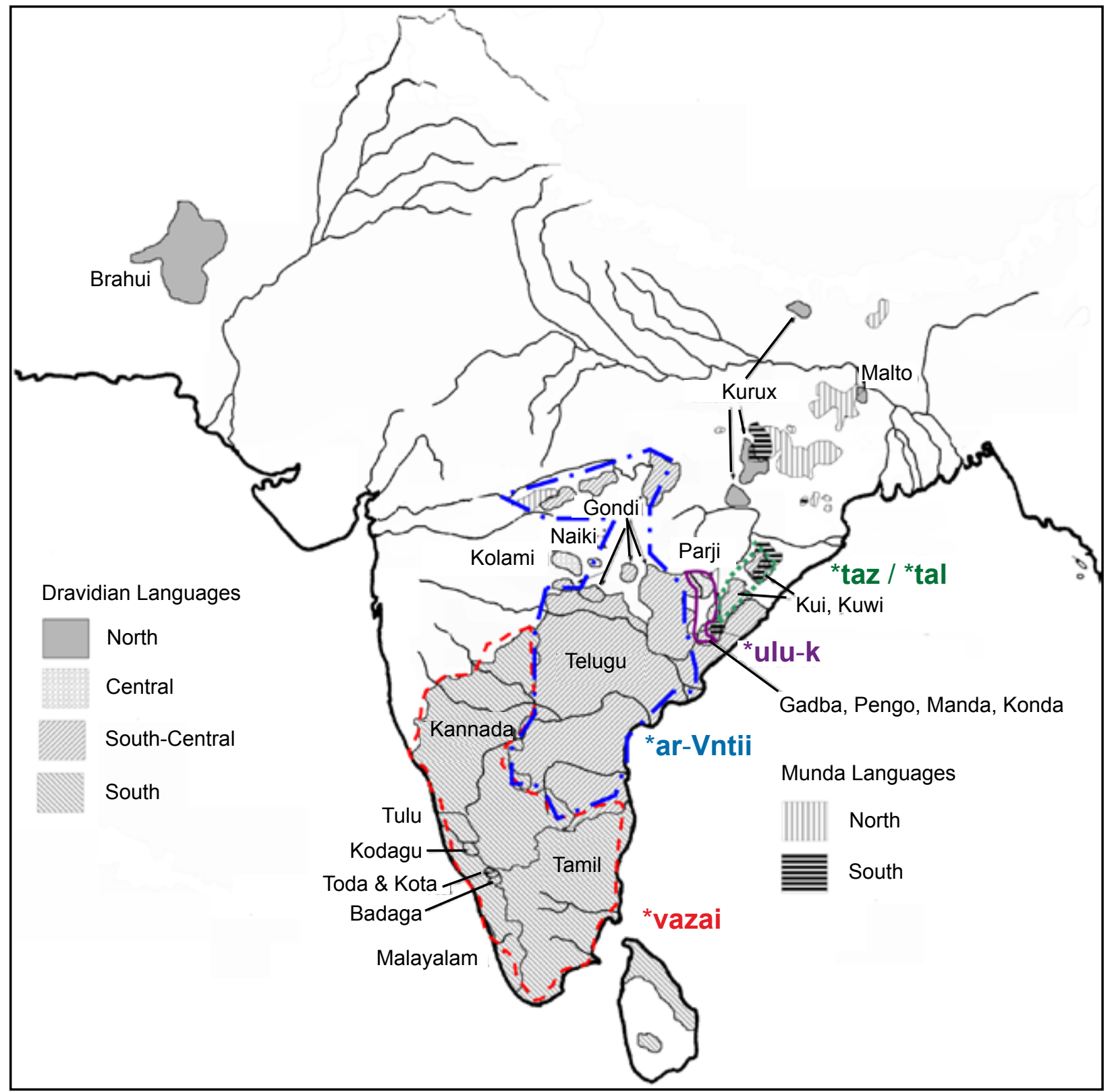

Figure 8. The Dravidian languages indicating the distribution of the 4 banana word roots. Also shown are the locations of Munda languages.

(Citrus, probably Mangifera) or across the Bay of Bengal (Santalum, Cocos, Areca), indicate the lines of cultural contact (trade) and diffusion which probably account for the arrival(s) of bananas in Dravidian-speaking southern India.

Indo-Aryan languages provide several banana terms congruent with multiple introductions or the introduction of multiple banana varieties. The botanical name Musa is taken from the Arabic mauz, which in turn derived from the Sanskrit mōcha (Yule \& Burnell 1886:715). This must be the same source for several languages of Nepal, e.g., Chepang maisai, Gurung mach, Magar mocha, Tamang moje, and Sunwar mugi (Manandhar 2002). The same source is reflected in a medieval Chinese name, mao-che (eighth c. A.D.) (Schafer 1967:186). This hints at dispersal of sweet bananas northwards from Assam through Yunnan. Other north Indian terms included Pali kadalī (Hindi kēla) (Rhys Davids \& Stede 1921-1925), undoubtedly the source of loanwords into several Munda languages (Mundari, Santali and Ho kadal; Juang kodilo) (Osada 2006), and several languages in Nepal, e.g., kera in Tharu, Newari, Danuwar, Bhojpuri, Mooshar (Manandhar 2002). The term kadalī can doubtless be derived from the series of Indonesian terms, of ultimate New Guinean origin, discussed by Donohue \& Denham (2009). This is presum- 
ably the same source as terms from South Dravidian(1) *wāz-a-, providing Tamil/Malayalam vārai and Tulu/Kannada bālè (Burrow \& Emeneau 1984), and presumably the classical pala of Pliny (Drury 1873:300). Achaya (1994:83) indicates that mōcha was for sweet forms while kadali was for cooking plantains, which also seems to be implied by related Dravidian terms. Nevertheless the unrelated terms in other Dravidian sub-groups may hint at an historical geography of several introductions to different parts of the Indian peninsula early in the period of the Bay of Bengal trade in the late first millennium B.C. or early in the first millennium A.D.

To place the above evidence in context, it is worth briefly summarizing the historical linguistics of South Asia. Excluding Nepal, South Asia is mainly host to three language families: Indo-Aryan (a branch of Indo-European), dominating the north and northwest; Dravidian, dominating the south with an outlier in Pakistan; and Munda (a branch of Austroasiatic) scattered in the hills of eastern and central India (Southworth 2005). Dravidian can be plausibly argued to have been in place somewhere in peninsular India since the start of the Neolithic or earlier (Fuller 2003, 2007 , Southworth 2005). By contrast the other groups are probable immigrants with agriculture during or after the Neolithic, with Indo-Aryan languages coming in from the northwest and Munda coming in from the northeast (but see Fuller 2007 for a less probable out-of-India hypothesis for Munda). While ancestral Munda-speaking groups are expected to have arrived with vocabulary for some crops from Southeast Asia, probably by c. 2000 B.C. (see Fuller 2003), there is no evidence for bananas in this vocabulary. The date for the earliest Indo-Aryan languages in the northwest is more controversial to date (see Bryant 2001), but can be broadly placed in the Late Early Bronze Age or Middle Bronze Age (broadly between 2500 and 1500 B.C.). These languages appear to have arisen to prominence after the Mature Harappan civilization (i.e., after 1900 B.C.) and to have been heavily influenced by one or more pre-existing languages, which have been interpreted as the Harappan language(s) (see Fuller 2007, Southworth 2005, Witzel 1999).

What should be emphasized, however, is that there is no clear linguistic trail for bananas that takes us back to the Harappan period Indus. Although the Harappan language(s) is/are extinct, and apparently not Dravidian (contra Parpola 1994), there remains an important substrate legacy, especially in terms relating to north/west Indian flora and agricultural crops (Fuller 2003, 2007, Southworth 2005, Witzel 1999), but we have no evidence for bananas among them. This may imply that the Harappan experiment with banana cultivation in Sindh vanished when that urban civilization collapsed. It is really only in the Sindh region where the evidence for Musa phytoliths occurs that the term "collapse" is really applicable to the transformations of the Late Harappan period (cf. Possehl 1997 ) and where aridification would have had the great- est impact (see Madella \& Fuller 2006). Thus banana cultivation in this region may have ceased with the Harappan collapse. It is also possible that Harappan cultivation was focused on producing a raw material such as paper or fiber rather than fruit, and the use of this may well have declined with urban collapse (including a possible loss of literacy in the Harappan sign system), such that it did not leave a lasting linguistic impression. In the latter case, a subsequent introduction of bananas for eating came with new loanwords from Southeast Asia into Indo-Aryan languages.

\section{Concluding Remarks}

India and China lie at the peripheries of the wild distribution of Musaceae and are unlikely to have contributed to the initial cultivation or early domestication processes of bananas. Nevertheless, complex later histories of introduction, probably by multiple routes and of multiple varieties, remain to be clarified. Thus far archaeobotanical evidence is very limited, but this is mainly a product of the absence of sampling, especially in regions of particular interest for bananas, such as Northeast India and southern China (from Yunnan to Guangdong). In addition, in other parts of China and India systematic phytolith studies, which might pick up past presence of bananas, are still few and far between.

The possibility of Harappan banana cultivation (25001900 B.C.) provides tantalizing hints that there may be some hidden prehistory of banana dispersal yet to be unearthed in some parts of South Asia, but the balance of evidence, especially from historical linguistics, suggests that the main introduction of edible bananas was some 2000 years later. This leads us to hypothesize that the Kot Diji Musa-type phytoliths may relate to cultivation of a Musaceae for raw material (fiber) or even ornamental purposes. This remains to be tested through finds of fiber. Nevertheless, existing linguistic data are imperfect. There is little understanding of the extent to which various indigenous terms relate to different Musa genome configurations ( $A A A, A B B$ and $A A B)$, to starchy or sweet varieties, and new linguistic and ethnobotanical data collection is needed in India. On the archaeological front, more systematic collection of phytolith data should gradually help to fill in the gaps in the knowledge of the past distribution of bananas in South Asia, East Asia and mainland Southeast Asia.

\section{Acknowledgements}

The authors are grateful to Qin Ling (School of Archaeology and Museology, Peking University) for assistance with Chinese sources. We thank Luc Vrydaghs, Tim Denham and Edmond De Lange, for useful comments on the original draft of this paper. 


\section{Fuller \& Madella - Banana Cultivation in South Asia and East Asia: A review 347 of the evidence from archaeology and linguistics}

\section{Literature Cited}

Achaya, K.T. 1994. Indian Food. A historical companion. Oxford University Press, Delhi.

Achaya, K.T. 1998. A Historical Dictionary of Indian Food. Oxford University Press, Delhi.

Ambasta, S.P., K. Ramachandran, K. Kashyapa \& R. Chand. 1986. Editors of The Useful Plants of India. Publications and Information Directorate, New Delhi.

Asouti, E. \& D.Q. Fuller. 2008. Trees and Woodlands in South India: An archaeological perspective. Left Coast Press, Walnut Creek.

Bowdery, D. 1999. Phytoliths from tropical sediments: reports from Southeast Asia and Papua New Guinea. Bulletin of the Indo-Pacific Prehistory Association 18:159-168.

Bryant, E. 2001. The Quest for the Origins of Vedic Culture: The Indo-Aryan migration debate. Oxford University Press, New York.

Chiu, H.-L., S.-I. Lee, C.-L. Yeh, C.-T. Shii \& C.-R. Yeh 2007. Musa balbisiana L. A. Colla, a newly naturalized wild banana in Taiwan. Journal of Taiwan Agricultural Research 56:215-223.

Desmond, R. 1992. The European Discovery of the Indian Flora. Oxford University Press, Oxford.

Donohue, M. \& T.P. Denham. 2009. Banana (Musa spp.) domestication in the Asia-Pacific region: Linguistic and archaeobotanical perspectives. Ethnobotany Research and Applications 7:293-332.

Drury, C.H. 1873 The Useful Plants of India with Notices of their Chief Value in Commerce, Medicine and the Arts. William Allan and Co, London.

Duthie, J.F. 1903 [reprint 1960]. Flora of the Upper Gangetic Plain. Botanical Survey of India, Calcutta.

Dykoski, C.A., R.L. Edwards, H. Cheng, D. Yuan, Y. Cai, M. Zhang, Y. Lin, J. Qing, Z. An \& J. Revenaugh 2005. A high-resolution absolute dated Holocene and deglacial Asian monsoon record from Dongge Cave, China. Earth and Planetary Science Letters 233:71-86.

Ellis, J.L. 1987. Flora of Nallamalais. 2 volumes. Botanical Survey of India, Calcutta.

Farmer, S.A., R. Sproat \& M. Witzel 2004. The collapse of the Indus-Script thesis: The myth of a literate Harappan civilization. Electronic Journal of Vedic Studies 11:19-57.
Fischer, C.E C. 1928. Flora of the Presidency of Madras. Volume III. Secretary of State for India, London.

Fischer, C.E.C. 1938. Where did the sandalwood tree (Santalum album Linn.) evolve? Journal of the Bombay Natural History Society 40:458-466.

Fuller, D.Q. 2003. An agricultural perspective on Dravidian historical linguistics: Archaeological crop packages, livestock and Dravidian crop vocabulary. Pp. 191-213 in Examining the Farming/Language Dispersal Hypothesis. Edited by P. Bellwood \& C. Renfrew. McDonald Institute for Archaeological Research, Cambridge.

Fuller, D.Q. 2006a. Agricultural origins and frontiers in South Asia: A working synthesis. Journal of World Prehistory 20:1-86.

Fuller, D. Q. 2006b. Silence before sedentism and the advent of cash-crops: A status report on early agriculture in South Asia from plant domestication to the development of political economies (with an excursus on the problem of semantic shift among millets and rice). Pp. 175-213 in Proceedings of the Pre-Symposium of RIHN and 7th ESCA Harvard- Kyoto Roundtable. Edited by T. Osada. Research Institute for Humanity and Nature, Kyoto.

Fuller, D.Q. 2007. Non-human genetics, agricultural origins and historical linguistics in South Asia. Pp. 393-443 in The Evolution and History of Human Populations in South Asia. Edited by M. Petraglia \& B. Allchin. Springer, Amsterdam.

Fuller, D.Q. 2008. Archaeological science in Field Training. Pp. 183-205 in From Concepts to Practical Strategies: The teaching of archaeological field techniques. Edited by P. J. Ucko, L. Qin \& J. Hubert. Saffron Press, London.

Fuller, D.Q., E. Harvey \& L. Qin. 2007. Presumed domestication? Evidence for wild rice cultivation and domestication in the fifth millennium $B C$ of the Lower Yangtze region. Antiquity 81:316-331.

Fuller, D.Q., R. Korisettar, P.C. Venkatasubbaiah \& M.K. Jones. 2004. Early plant domestications in southern India: Some preliminary archaeobotanical results. Vegetation History and Archaeobotany 13:115-129.

Fuller, D.Q. \& M. Madella. 2001. Issues in Harappan archaeobotany: Retrospect and prospect. Pp. 317-390 in Indian Archaeology in Retrospect. Volume II. Protohistory. Edited by S. Settar \& R. Korisettar. Publications of the Indian Council for Historical Research. Manohar, New Dehli.

Fuller, D.Q., L. Qin \& E. Harvey. 2008. Rice archaeobotany revisited: Comments on Liu et al. (2007). Antiquity 82:315. On-line project gallery. 
Fuller, D.Q, L. Qin, Y. Zheng, Z. Zhao, X. Chen, L.A. Hosoya $\&$ G.-P. Sun 2009 . The domestication process and domestication rate in rice: Spikelet bases from the Lower Yangtze. Science 323:1607-1610.

Fuller, D.Q. \& H. Zhang. 2007. A preliminary report of the survey archaeobotany of the upper Ying Valley (Henan Province). Pp. 916-958 in Dengfeng wangchenggang yizhi de faxian yu yanjiu (2002-2005) [ Archaeological Discovery and Research at the Wangchenggang Site in Dengfeng (2002-2005)]. Edited by School of Archaeology and Museology, Peking University and Henan Provincial Institute of Cultural Relics and Archaeology. Great Elephant Publisher, Zhengzhou.

Haines, H.H. 1921-1925. Botany of Bihar and Orissa. Adlard and Newman, London.

Harbaugh, D.T. \& B.G. Baldwin. 2007. Phylogeny and biogeography of the sandalwoods (Santalum, Santalaceae): repeated dispersals throughout the Pacific. Annals of Botany 94:1028-1040.

Harvey, E.L. 2006. Early Agricultural Communities in Northern and Eastern India: An archaeobotanical investigation. Ph.D. Dissertation, Institute of Archaeology, University College London.

Harvey, E.L. \& D.Q. Fuller. 2005. Investigating crop processing through phytolith analysis: The case of rice and millets. Journal of Archaeological Science 32:739-752.

Harvey, E.L., D.Q. Fuller, K.K. Basa, R. Mohany \& B. Mohanta. 2006. Early Agriculture in Orissa: Some archaeobotanical results and field observations on the Neolithic. Man and Environment 31:21-32.

Harvey, E.L., D.Q. Fuller, J.N. Pal \& M.C. Gupta. 2005. Early agriculture of Neolithic Vindyhas (North-Central India). Pp. 329-334 in South Asian Archaeology 2003. Proceedings of the European Association for South Asian Archaeology Conference, Bonn, Germany, $7^{\text {th }}-11^{\text {th }}$ July 2003. Edited by U. Franke-Vogt \& J. Weisshaar. Linden Soft, Aachen.

Ho, P.-t. 1977. The indigenous origins of Chinese agriculture. Pp. 413-484 in The Origins of Agriculture. Edited by C. A. Reed. Mouton, The Hague and Paris.

Hooker, J.D. 1872-1897. The Flora of British India. 7 volumes. Reeve and Co, London.

Huang, F. \& M. Zhang 2000. Pollen and phytolith evidence for rice cultivation during the Neolithic at Longqiuzhuang, eastern Jianghuai, China. Vegetation History and Archaeobotany 9:161-168.
ICAR [Indian Council for Agricultural Research] 1980. Handbook of Agriculture. Revised Edition. Indian Council for Agricultural Research, Delhi.

Jiang, Q. 1995. Searching for evidence of early rice agriculture at prehistoric sites in China through phytolith analysis: an example from central China. Review of Palaeobotany and Palynology 89:481-485

Jin, G. 2006. Phytolith analysis for the archaeological sites of pre-Qin dynasties in Shandong and some related questions. Dongfang Kaogu [Oriental Archaeology] 3:258-279.

Jin, G. 2008. Phytolithic Analysis and Study of Pre-Qin Sites of Shandong (1997-2003). Pp. 20-40 in Studies of Early Agriculture and Anthropology in Shandong. Edited by F. Luan \& K. Miyamoto. Science Press, Beijing.

Jin, G.Y., S.D. Yan, T. Udatsu, Y.F. Lan, C.Y. Wang \& P.H. Tong. 2007. Neolithic rice paddy from the Zhaojiazhuang site, Shandong, China. Chinese Science Bulletin 52:33763384.

Jin, G. \& C.Y. Wang. 2007. Analysis of phytoliths samples from the survey of the Upper and Mid Ying River Valley. Pp. 805-814 in Dengfeng wangchenggang yizhi de faxian yu yanjiu (2002-2005) [Archaeological Discovery and Research at the Wangchenggang Site in Dengfeng (20022005)]. Edited by School of Archaeology and Museology, Peking University and Henan Provincial Institute of Cultural Relics and Archaeology. Great Elephant Publisher, Zhengzhou.

Kajale, M.D. 1989. Mesolithic exploitation of wild plants in Sri Lanka: Archaeobotanical study at the cave site of BeliLena. Pp. 269-281 in Foraging and Farming: The evolution of plant exploitation. Edited by D.R. Harris \& G.C. Hillman. Routledge, London.

Kajale, M.D. 1996. Palaeobotanical investigations at Balathal: Preliminary results. Man and Environment 21:98102.

Kajale, M.D. \& S.P. Eksambekar 1997. Application of phytolith analyses to a Neolithic site at Budihal, Distirct Gulbarga, South India. Pp. 219-229 in Estado Actual de Los Etudios de Fitolitos en Suelos y Plantas (The State of the Art of Phytoliths in Soils and Plants). Edited by A. Pinilla, J. Juan-Tresserras \& M.J. Machado. Consejo Superior de Investigaciones Cientificas (CSIC), Madrid.

Kajale, M.D. \& S.P. Eksambekar. 2001. Phytolith approach for investigating ancient occupations at Balathal, Rajasthan, India. Pp. 199-212 in Phytoliths: Application in earth sciences and human history. Edited by J.D. Maunier \& F. Colin. A.A. Balkema, Lisse.

Kajale, M.D. \& S.P. Eksambekar 2007. Phytolith analytical study on a late Chalcolithic - early historical archaeo- 


\section{Fuller \& Madella - Banana Cultivation in South Asia and East Asia: A review 349 of the evidence from archaeology and linguistics}

stratigraphical sequence from Balathal (south Rajasthan, India). Pp. 118-133 in Plants, People and Places - Recent studies in phytolith analysis. Oxbow Monograph. Edited by M. Madella \& D. Zurro. Oxbow Books, Oxford.

Karlgren, B. 1923. Analytic Dictionary of Chinese and Sino-Japanese. Librairie Orientaliste Paul Guethner, Paris.

Kealhofer, L. \& D.R. Piperno 1996. The phytoliths sequence from the Bang Pakong Valley. Pp. 227-238 in The Excavations of Khok Phanom Di, a Prehistoric Site in Central Thailand. Volume IV. Subsistence and Environment: The botanical evidence. The Biological Remains Part III. Edited by G.B. Thompson.The Society of Antiquaries of London, London.

Lee, G.-A., G.A. Crawford, L. Liu \& X. Chan. 2007. Plants and people from the early Neolithic to Shang periods in North China. Proceedings of the National Academy of Sciences (USA) 104:1087-1092.

Lu H.Y. \& J.P. Zhang. 2008. Neolithic cultural evolution and Holocene climate change. Quaternary Sciences 28:1050-1060.

Lu, T.L.D. 2006. The Occurrence of Cereal Cultivation in China. Asian Perspectives 45:129-158.

Lu, T.L.D., Z. Zhao \& Z. Zheng 2005. The prehistoric and historic environments, vegetations and subsistence strategies at Sha Ha, Sai Kung. Pp. 57-63 in The Ancient Culture of Hong Kong. Archaeological discoveries in Sha Ha, Sai Kung. Edited by Antiquites and Monuments Office. Leisure and Culture Services Department, Hong Kong.

MacKay, E.J.H. 1938. Further Excavations at Mohenjodaro. 2 volumes. Government of India, New Delhi.

Madella, M. 2003. Investigating agriculture and environment in South Asia: Present and future contributions from opal phytoliths. Pp. 199-249 in Indus Ethnobiology: New perspectives from the field. Edited by S. Weber \& W. Belcher. Lexington Books (Rowman \& Littlefield Publishing Group), Lanham.

Madella, M. \& D.Q. Fuller 2006. Paleoecology and the Harappan civilisation of South Asia: A reconsideration. Quaternary Science Reviews 25:1283-1301.

Madella, M., D.Q. Fuller, N. Boivin \& R. Korisettar. n.d.a. Unpublished phytolith data from Sanganakallu and Kupgal ashmound sites.

Madella, M., R. Korisettar \& U. Rajala. n.d.b. Ongoing research on Ashmound Tradition sites in Karnataka and Andra Pradesh. Unpublished data.

Manandar, N.P. 2002. Plants and People of Nepal. Timber Press, Portland.
Marshall, J. 1931. Mohenjo-daro and the Indus Civilization. 3 volumes. A Probsthain, London.

Matthew, K.M. 1995. An Excursion Flora of Central Tamil Nadu, India. Oxford \& IBH, New Delhi.

Mbida, Ch., E. De Langhe, L. Vrydaghs, H. Doutrelpont, R. Swennen, W. Van Neer \& P. de Maret. 2006. Phytolith evidence for the early presence of domesticated banana (Musa) in Africa. Pp. 68-81 in Documenting Domestication: New Genetic and Archaeological Paradigms. Edited by M. Zeder, D. Bailer, E. Emshwiller \& B.D. Smith. University of California Press, Berkeley.

Mbida, Ch., M.W. Van Neer, H. Dotrelepont \& L. Vrydaghs 2000. Evidence for banana cultivation and animal husbandry during the first millennium $B C$ in the forest of Southern Cameroon. Journal of Archaeological Science 27:151-162.

McKean, M.B. 1983. The Palynology of Balakot, a preHarappan and Harappan age site in Las Bela, Pakistan. Ph.D. Dissertation, Southern Methodist University. Ann Arbor University Microfilms.

Osada, T. 2006. How many Proto-Munda words in Sanskrit? With special reference to agricultural vocabulary. Pp. 151-174 in Proceedings of the Pre-Symposium of RIHN and $7^{\text {th }}$ ESCA Harvard-Kyoto Roundtable. Edited by T. Osada \& N. Hase. Research Institute for Humanity and Nature, Kyoto.

Possehl, G.L. 1997. The transformation of the Indus civilisation. Journal of World Prehistory 11:425-472.

Prasad, S. \& Y. Enzel. 2006. Holocene paleoclimates of India. Quaternary Research 66:442-453.

Rahaman, M. 1993. Land and Life in Sindh - Pakistan. Ferozsons, Lahore.

Ren, G. \& H.-J. Beug. 2002. Mapping Holocene pollen data and vegetation of China. Quaternary Science Reviews 21:1395-1422.

Reynolds, P.K. \& C.Y. Fang. 1940. The banana in Chinese literature. Harvard Journal of Asiatic Studies 5:165-181.

Rhys Davids, T.W. \& W. Stede. 1921-1925. The Pali Text Society's Pali-English Dictionary. Pali Text Society, London.

Saraswat, K.S. 2005. Agricultural background of the early farming communities in the Middle Ganga Plain. Pragdhara (Journal of the Uttar Pradesh State Department of Archaeology) 15:145-177.

Schafer, E.H. 1967. The Vermillion Bird. T'ang images of the South. University of California Press, Berkeley. 
Sharma, B.D., S. Karthikeyan \& N.P. Singh. 1996. Flora of Maharashtra State - Monocotyledons. Botanical Survey of India, Calcutta.

Sharma, B.D., N.P. Singh, R.S. Raghavan \& U.R. Deshpande. 1984. Flora of Karnataka. Botanical Survey of India, Calcutta.

Sharma, G.R., V.D. Misra, D. Mandal, B.B. Misra \& J.N. Pal. 1980. Beginnings of Agriculture (Epi-Palaeolithic to Neolithic: Excavations at Chopani-Mando, Mahadaha, and Mahagara). Allahabad, Abinash Prakashan.

Sharma, P.P. \& N.P. Singh. 2001. Ethnobotany of Dadra, Nagar Haveli and Daman (Union Territories). Botanical Survey of India, Calcutta.

Shaw, J., J. Sutcliffe, L. Lloyd-Smith, J.-L. Schwenninger, M. Chahuan, O. Misra \& E. Harvey. 2007. Ancient irrigation and Buddhist history in Central India: Optically stimulated luminescence dates and pollen sequences from Sanchi Dams. Asian Perspectives 46:166-201.

Shetty, B.V. \& V. Singh. 1991. Flora of Rajasthan. 3 volumes. Botanical Survey of India, Calcutta.

Simmonds, N.W. 1962. The Evolution of Bananas. Longman, London.

Simmonds, N.W. 1995. Bananas. Pp. 370-375 in Evolution of Crop Plants. Second edition. Edited by J. Smartt \& N.W. Simmonds. Longman Scientific and Technical, Essex.

Singh, N.P., K.K. Khanna, V. Mudgal \& R.D. Dixit. 2001. Flora of Madhya Pradesh. Volume III. Botanical Survey of India, Calcutta.

Southworth, F.C. 2005. The Linguistic Archaeology of South Asia. Routledge, London.

Southworth, F.C. 2006. Proto-Dravidian agriculture. Pp. 121-150 in Proceedings of the Pre-Symposium of RIHN and $7^{\text {th }}$ ESCA Harvard - Kyoto Roundtable. Edited by T. Osada \& N. Hase. Research Institute for Humanity and Nature, Kyoto.

Wang, D., Z.Q. Yao, C.S. Wang, Y.W. Hu, G.Q. Chen \& Q.C. Zhang 2007. The phytolith analysis of Shangjifangyingzi site. Nongye Kaogu [Agricultural Archaeology] 20071:48-55.

Weber, S.A. 1999. Seeds of urbanism: paleoethnobotany and the Indus civilization. Antiquity 73:813-826.

Weber, S.A. 2003. Archaeobotany at Harappa: Indications for change. Pp. 175-198 in Indus Ethnobiology: New perspectives from the field. Edited by S. Weber \&W. Belcher.
Lexington Books (Rowman \& Littlefield Publishing Group), Lanham.

Weiskopf, A. 2005. Investigation of the Neolithic Ashmound and Settlement of Sanganakallu in the South Deccan, India using phytoliths and macro-archaeobotanical materials. M.Sc. Dissertation. Institute of Archaeology, University College London, London.

Wilson, S.M. 1985. Phytolith analysis at Kuk, an Early Agricultural site in Papua, New Guinea. Archaeology in Oceania 20:90-97

Witzel, M. 1999. Early sources for South Asian substrate languages. Mother Tongue: Special Issue 1-76.

Wu, D. \& W.J. Kress. 2000. Musaceae. Pp. 297-318 in Flora of China. Vol. 24 (Flagellariaceae through Marantaceae). Edited by Zheng Yi Wu \& Peter H. Raven. Science Press, Bejing and Missouri Botanical Garden Press, St. Louis.

Yule, H. \& A.C. Burnell. 1886 [1996]. Hobson-Jobson. The Anglo-Indian Dictionary. Wordsworth Editions, London.

Zhao, Z.J. 1995. Analysis of phytoliths from Xian Ren Dong and Wang Dong, in origins of rice agriculture. Pp. 47-52 in The Preliminary Report of the Sino-American Jiangxi (PRC) Project SAJOR. Edited by R. MacNeish \& J.G. Libby. El Paso Centennial Museum, The University of Texas, El Paso.

Zhao, Z.J. 1998. The Middle Yangtze region in China is one place where rice was domesticated: Phytolith evidence from the Diaotonghuan Cave, Northern Jaingxi. Antiquity 72:885-897.

Zhao, Z.J. 2003. Unearthed plant remains from Zengpiyan site and their analysis. Pp. 286-296 in The Zengpiyan Cave in Guilin. Edited by Institute-of-Archaeology(CASS), Guangxi-Archaelogical-Team, Zengpiyan-Museum \& Guilin-Archaeological-Team. Cultural Relics Publishing House, Beijing.

Zhao, Z. 2008. Flotation techniques and their application in Chinese archaeology. Pp. 207-212 in From Concepts to Practical Strategies: The teaching of archaeological field techniques. Edited by P.J. Ucko, L. Qin \& J. Hubert. Saffron Press, London.

Zhao, Z. \& D. Piperno. 2000. Late Pleistocene/Holocene environments in the Middle Yangtze River Valley, China and rice (Oryza sativa L.) domestication: The phytoliths evidence. Geoarchaeology 15:203-222.

Zheng, Y., A. Matsui, \& H. Fujiwara. 2004. Phytoliths of rice detected in the Neolithic sites in the Valley of the Taihu Lake in China. Environmental Archaeology 8:177-183. 


\section{Fuller \& Madella - Banana Cultivation in South Asia and East Asia: A review 351 of the evidence from archaeology and linguistics}

Zheng, Y., G. Sun \& X. Chen. 2007. Characteristics of the short rachillae of rice from archaeological sites dating to 7000 years ago. Chinese Science Bulletin 52:1654-1660.

Zombrich, T.J. 2007. The origin and diffusion of betel chewing: A synthesis of evidence from South Asia, Southeast Asia and beyond. Electronic Journal of Indian Medicine 1:63-116
Zou, H., J. Gu, M. Li, L. Tang, J. Ding \& Q. Yao. 2000. Findings of paddies of Majiabang Culture at Caoxieshan, Jiangsu Province. Pp. 97-114 in The Origins of Rice Agriculture, Pottery and Cities. Edited by W. Yan \& Y. Yasuda. Cultural Relics Publishing House, Beijing. 
